\title{
Elektronische Wedhselwirkung zwischen aufgedampften Germaniumfilmen und Sauerstoff
}

\author{
Von R. Suhrmann, M. Kruel ${ }^{1}$ und G. Wedler \\ Aus dem Institut für Physikalische Chemie und Elektrochemie der Technischen Hochschule Hannover \\ (Z. Naturforschg. 18 a, 119-125 [1963]; eingegangen am 22. November 1962)
}

Herrn Professor Dr. Dr. h.c. KLaus Cuusius zum 60. Geburtstag gewidmet

\begin{abstract}
Ge films of thicknesses within the p-boundary layer thickness are precipitated under ultra high vacuum conditions, at $77{ }^{\circ} \mathrm{K}$. They are investigated either in the disordered state (before annealing at a higher temperature) or in the ordered state (after annealing). The electric resistance $R$ and the photoelectric work function $\Phi$ are measured before and after the influence of known amounts of oxygen. $R$ is plotted as a function of time and coverage $n, \Phi$ as a function of $n$. Small amounts of oxygen cause $R$ to increase without changing $\Phi$, large amounts result in a succeeding decrease of $R$ and cause $\Phi$ to increase by 0.1 to $0.2 \mathrm{~V}$. The results are discussed by means of a band model. At small coverages oxygen acts as an electron donator, at higher coverages as an electron acceptor.
\end{abstract}

Das Studium der elektronischen Wechselwirkung zwischen adsorbierten Gasen und Germanium ist von besonderem Interesse, weil im Germanium ein elementarer Halbleiter vorliegt, für dessen elektrischen Oberflächenaufbau experimentell gut gestützte theoretische Vorstellungen vorhanden sind, so daß die bei der Chemisorption beobachteten Änderungen von elektrischer Leitfähigkeit und Elektronen-Austrittspotential am ehesten gedeutet werden können. Aus diesem Grunde wird in der vorliegenden Arbeit der Einfluß der Adsorption von Sauerstoff und in einer anschließenden ${ }^{2}$ der von Kohlenmonoxyd auf die genannten elektronischen Eigenschaften von GeFilmen studiert.

Der Einfluß adsorbierten Sauerstoffs auf den elektrischen Widerstand und das Elektronen-Austrittspotential von Germanium ist bisher nur an Kristallen untersucht worden. Entsprechende Versuche an im Ultra-Hochvakuum aufgedampften GeFilmen mit reiner Oberfläche liegen bisher nicht vor, obwohl sie besonders dann zu aufschlußreichen Ergebnissen führen sollten, wenn die Schichtdicke der Filme innerhalb der Dicke der p-Randschicht liegt $(<1000 \AA)$.

Das Widerstands-Temperaturverhalten solcher Filme ergibt $^{3}$ zwei Aktivierungsenergien von 0,1 und $0,2 \mathrm{eV}$, die wegen der p-Leitung der Filme als Abstände von Akzeptorniveaus von der Valenzband-

1 Siehe auch M. KRUel, Dissertation, Technische Hochschule Hannover 1961.

2 Diese Arbeit erscheint demnächst.

3 R. Suhrmann, M. Kruel u. G. Wedler, Z. Phys., im Druck.

${ }^{4}$ z. B. R. Suhrmann u. G. Wedler, Z. angew. Phys. 14, 70 [1962]. kante anzusehen sind. Während aufgedampfte reine Metallfilme $^{4}$ nur ein einziges Austrittspotential aufweisen, besitzt die Oberfläche von Ge-Filmen zwei Austrittspotentiale ${ }^{3,5} \quad \Phi_{1}$ und $\Phi_{2}$ von etwa 5,0 bzw. 5,1 Volt. Die zugehörigen Mengenkonstanten ${ }^{6}$ $(\alpha \cdot M)_{1}$ und $(\alpha \cdot M)_{2}$ zeigen eine deutliche Abhängigkeit von der Meßtemperatur, und zwar derart, daß die zum kleineren Austrittspotential $\Phi_{1}$ (höherem Akzeptorniveau) gehörende Mengenkonstante $(\alpha \cdot M)_{1}$ mit zunehmender Temperatur gegenüber $(\alpha \cdot M)_{2}$ anwächst. Diese Befunde lassen sich durch das in Abb. 1 wiedergegebene Bändermodell eines Ge-Films darstellen.

\section{Experimentelles}

Abb. 2 zeigt das Blockdiagramm der verwendeten Ultra-Hochvakuumapparatur, mit der unter Berücksichtigung der üblichen Maßnahmen ein Vakuum von etwa $2 \cdot 10^{-10}$ Torr erreicht wurde. Einzelheiten der verwendeten Meßzelle sind aus Abb. 3 zu ersehen. Sie war bei den Messungen mit einer geerdeten Hydrokollagschicht bestrichen.

Die Ge-Filme wurden bei $77^{\circ} \mathrm{K}$ aufgedampft und entweder ohne vorherige Temperung (im ungeordneten Zustand) untersucht oder sie wurden nach dem Aufdampfen eine Stunde lang bei 293 oder $373^{\circ} \mathrm{K}$ getempert und anschließend in einem geordneteren Zustand studiert. Durch das Tempern nahm der Widerstand stark zu, da die als Akzeptoren wirkenden Gitterstörun-

5 R. Suhrmann u. L. van Lessen, Abh. Braunschw. Wiss. Gesellsch. 10, 35 [1958].

${ }^{6}$ Der Faktor $\alpha$ in der Mengenkonstanten berücksichtigt die Wechselwirkungs-Wahrscheinlichkeit zwischen Photon und Elektron. 

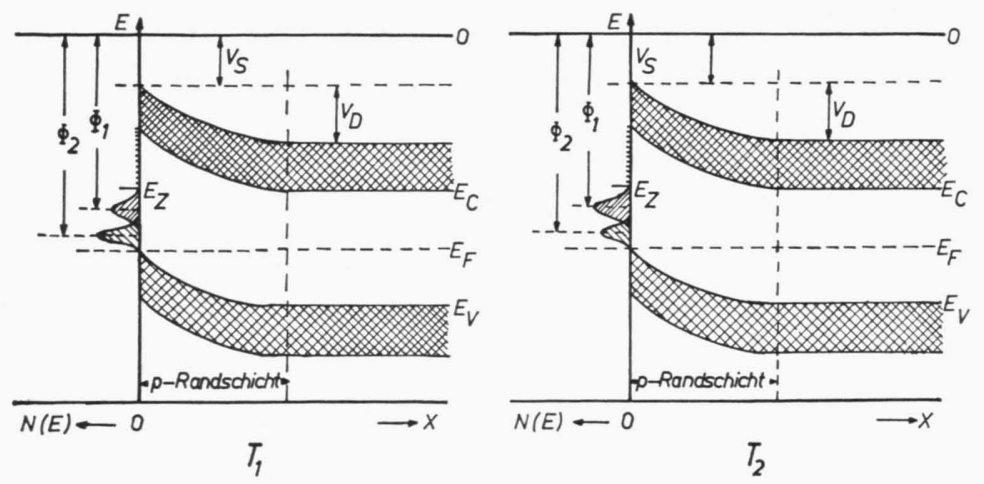

Abb. 1. Bändermodell eines Ge-Films bei tiefer $\left(T_{1}\right)$ und höherer $\left(T_{2}\right)$ Temperatur. Die einfach schraffierten Flächen geben die Besetzungswahrscheinlichkeit $N(E)$ der Oberflächenzustände an. $V_{\mathrm{S}}=$ durch die einseitige Polarisierung der obersten Ge-Atomlage entstehender Potentialsprung, $V_{\mathrm{D}}=$ Diffusionsspannung, $E_{Z}=$ höchstes Niveau der besetzten Oberflächenzustände (fast states), $E_{\mathrm{C}}=$ untere Kante des Leitungsbandes, $\quad E_{\mathrm{F}}=\mathrm{F}_{\mathrm{ER} M I}-$ Niveau, $\quad E_{\mathrm{V}}=$ obere Kante des Valenzbandes; $x=0$ Oberfläche, $\Phi_{1}$ und $\Phi_{2}=$ photoelektrische Austrittspotentiale.
$T_{2}>T_{1}$

gen der p-leitenden Filme zum Teil ausheilten. Die Messungen von Widerstand, Austrittspotential und adsorbierter Menge Sauerstoff erfolgten bei $77^{\circ}$ und $273^{\circ} \mathrm{K}$.

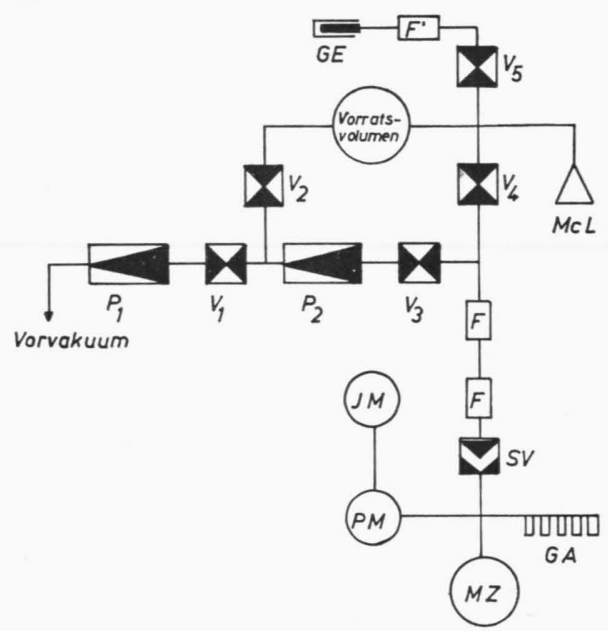

Abb. 2. Blockdiagramm der Ultrahochvakuum-Apparatur. $\mathrm{P}$ dreistufige Quecksilberpumpen, V manometrische Quecksilberverschlüsse, SV ungefetteter Kugelschliff-Verschluß, F Kühlfallen, McL McLeodsches Manometer, IM Ionisationsmanometer vom Alpert-Typ, PM Pirani-Manometer, MZ Meßzelle (s. Abb. 3), GE Gasentwicklung für Sauerstoff, GA Zertrümmerungs-Ampullen.

Der Widerstand der Filme $\left(10^{6}\right.$ bis $\left.10^{12} \Omega\right)$ wurde nach einer Kompensationsmethode bestimmt, und zwar bis auf $0,1 \%$ direkt und bis $0,01 \%$ durch Interpolation der Ausschläge am Meßinstrument des Verstärkers.

Die Bestimmung des Elektronen-Austrittspotentials $\Phi$ erfolgte durch Ermittlung der spektralen Ausbeute an Photoelektronen und Anwendung der Fowlenschen Theorie $^{4,7}$. Die Lichtverluste im Quarzfenster der Meßzelle wurden berücksichtigt.

7 H. Simon u. R. Suhrmann, Der lichtelektrische Effekt und seine Anwendungen, Springer-Verlag, Berlin, Göttingen, Heidelberg 1958.
Zum Aufdampfen diente Germanium der Firma Johnson, Matthey \& Co., London; in ihm waren $\mathrm{Ca}, \mathrm{Cu}, \mathrm{Mg}$ und $\mathrm{Ag}$ in einer Konzentration von $<1$ p.p.m. enthalten, entsprechend einer Verunreinigung von ca. $10^{16}$ Atomen pro $\mathrm{cm}^{3}$; bezüglich anderer metallischer Verunreinigungen war das Germanium spektralrein; vor dem Aufdampfen aus der Wolframwendel wurde es im Ultra-Hochvakuum in der Wendel vorsichtig geschmolzen und entgast. Zur Bestimmung der Schichtdicke $d$ wurden die Filme in Salpetersäure

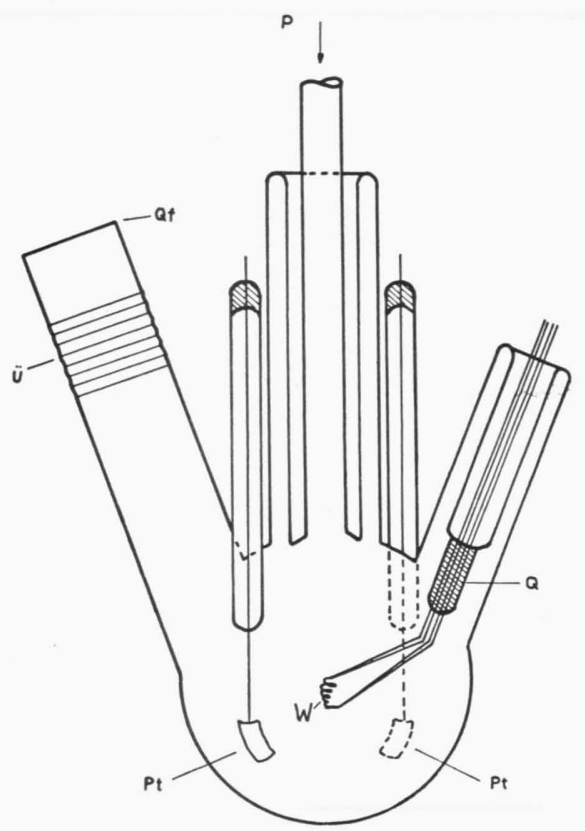

Abb. 3. Meßzelle für gleichzeitige Messung von Widerstand und lichtelektrischer Empfindlichkeit. W Wolframwendel mit Germanium (gleichzeitig Anode für den Photoelektronenstrom), Q Quetschfuß mit 4 Stromzuführungen, Pt PlatinKontaktfolien zur Messung des Filmwiderstandes (Kathodenzuführungen für den Photoelektronenstrom), Qf Quarzfenster, U Übergangsstück Quarz/Duran 50, P Gaseintrittsrohr mit Pumpleitung. 
gelöst und als $\mathrm{GeO}_{2}$ ausgewogen; $d$ lag im allgemeinen zwischen 300 und $900 \AA$.

Der Sauerstoff wurde durch thermische Zersetzung von $\mathrm{KMnO}_{4}$ gewonnen.

\section{Ergebnisse}

Die Abb. 4, 5 und 6 zeigen den zeitlichen Verlauf der Einwirkung von Sauerstoff auf den elektrischen Widerstand $R$ (Teilbild a) und das Austrittspotential $\Phi_{1}$ (Teilbild d) ${ }^{8}$ von Ge-Filmen verschiedenen Ordnungszustandes und verschiedener Schichtdicke. Gleichzeitig ist aus Teilbild b der $\mathrm{O}_{2}$-Druck in der

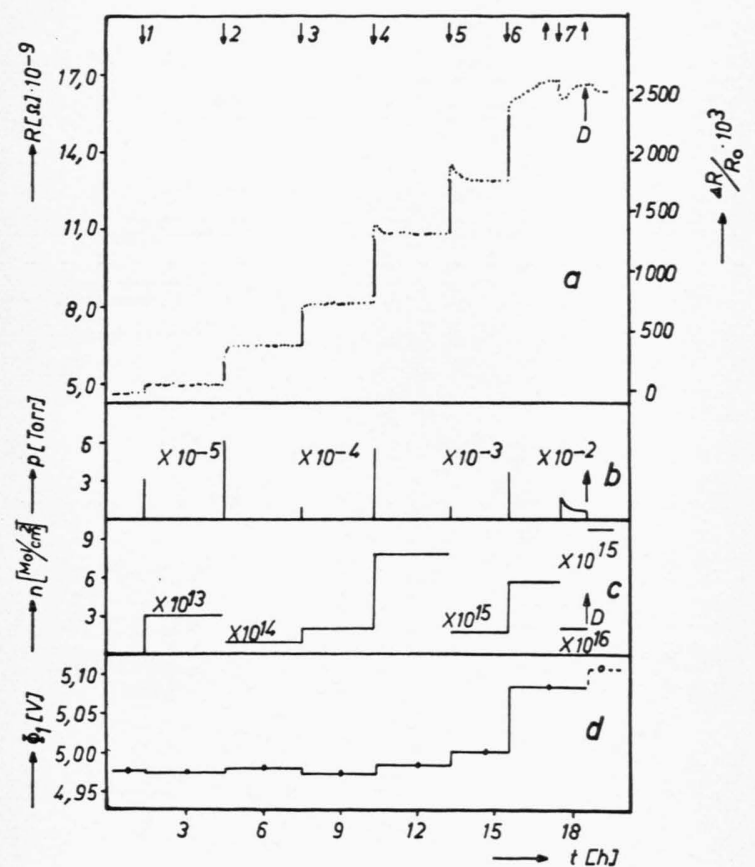

Abb. 4. Einwirkung von Sauerstoff bei $77^{\circ} \mathrm{K}$ auf einen ungeordneten (nicht getemperten) Ge-Film von 350 A Schichtdicke. a) Widerstand $R$ und relative Widerstandsänderung $\left.\Delta R / R_{0} \cdot 10^{3}, \mathrm{~b}\right)$ Druck $\left.p, \mathrm{c}\right)$ Belegung $n$ (Molekeln $\mathrm{O}_{2}$ pro $\mathrm{cm}^{2}$ ), d) Austrittspotential $\Phi_{1}$. Nach unten gerichtete Pfeile bedeuten: Gaszugabe, nach oben gerichtete: Abpumpen des Gases. $R_{0}$ Anfangswiderstand.

Gasphase und aus c die daraus berechnete Belegung in Molekeln pro $\mathrm{cm}^{2}$ geometrischer Oberfläche $\mathrm{zu}$ entnehmen. Tritt eine momentane Adsorption ein, so gibt die Höhe des Peaks in b den Druck an, der sich ohne Adsorption des zugegebenen Gases hätte einstellen müssen.

8 Die Meßgenauigkeit von $\Phi_{1}$ ist wesentlich größer als die von $\Phi_{2}$, so daß die Änderungen von $\Phi_{1}$ außerhalb, die

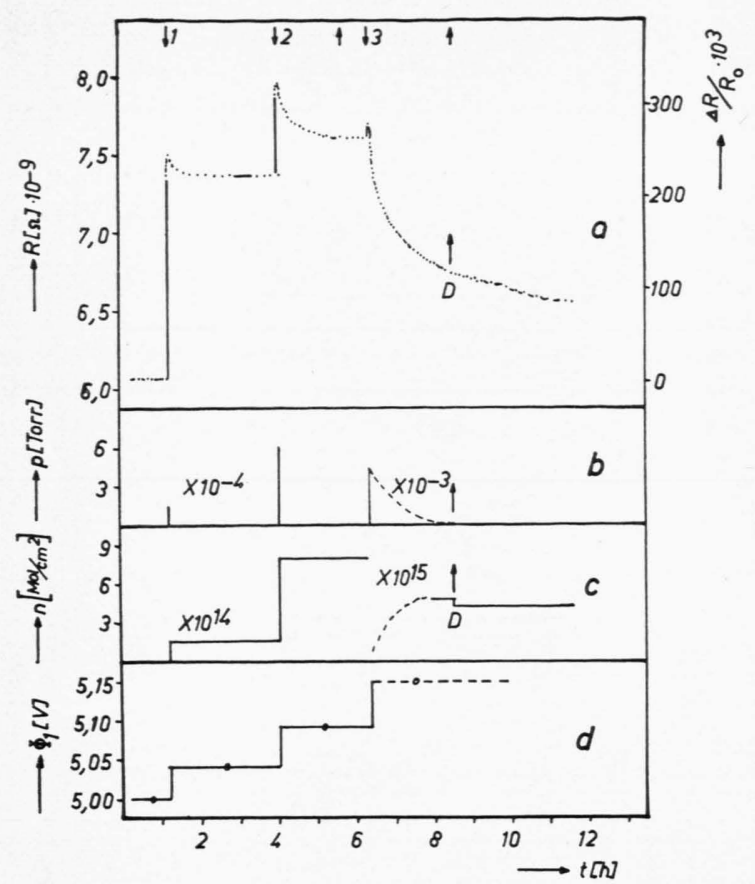

Abb. 5. Einwirkung von Sauerstoff bei $77^{\circ} \mathrm{K}$ auf einen bei $293{ }^{\circ} \mathrm{K}$ getemperten Ge-Film von $880 \AA$ Schichtdicke.

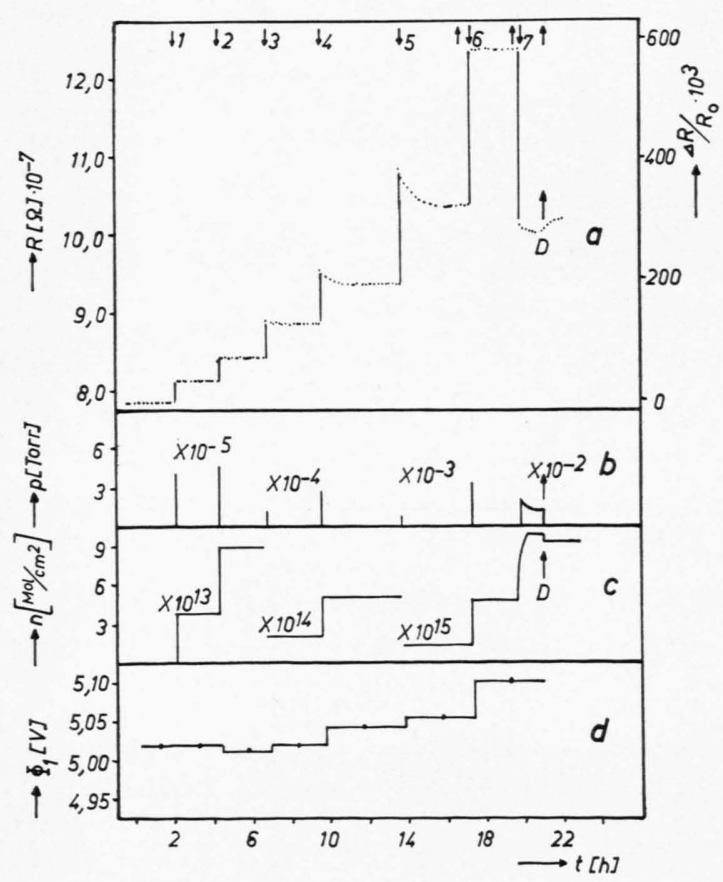

Abb. 6. Einwirkung von Sauerstoff bei $273{ }^{\circ} \mathrm{K}$ auf einen bei $373^{\circ} \mathrm{K}$ getemperten Ge-Film von 830 Å Schichtdicke.

von $\Phi_{2}$ jedoch häufig innerhalb der Meßfehlergrenze liegen. 
Bei niedrigen Belegungen tritt stets eine beträchtliche Zunahme des Widerstandes auf. Bei höheren Belegungen überlagert sich - insbesondere bei den

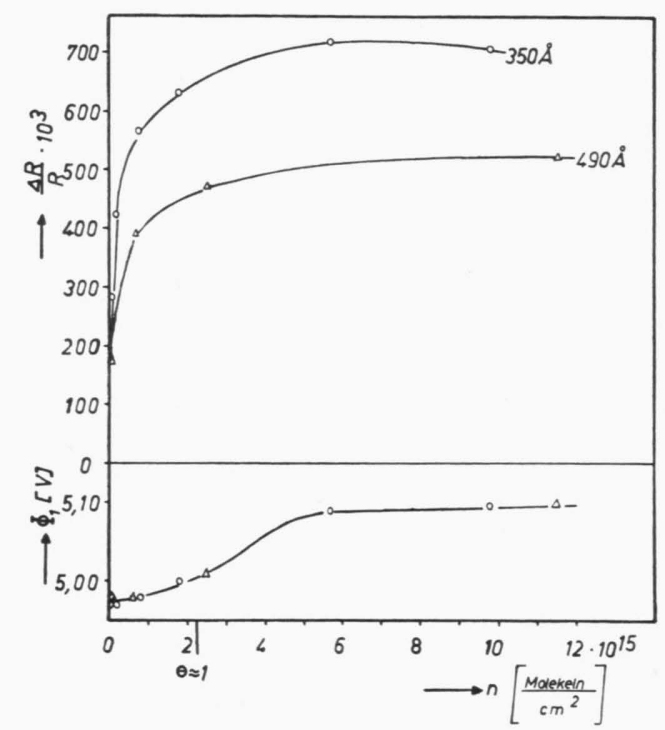

Abb. 7. Abhängigkeit der relativen Widerstandsänderung $\Delta R / R$ und des Austrittspotentials $\Phi_{1}$ von der Belegung verschieden dicker ungeordneter Ge-Filme bei $77^{\circ} \mathrm{K}$. $R$ jeweiliger Endwiderstand nach Zugabe des Gases.

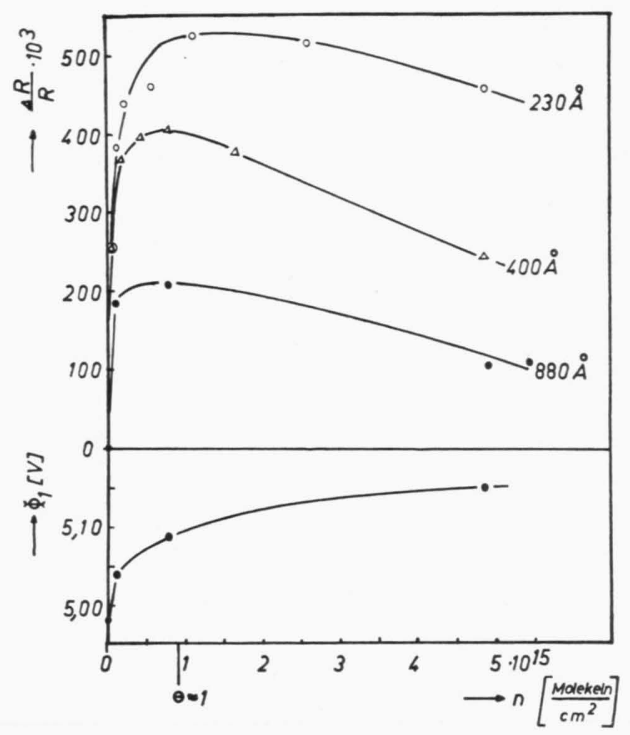

Abb. 8. Abhängigkeit der relativen Widerstandsänderung $\Delta R / R$ und des Austrittspotentials $\Phi_{1}$ von der Belegung verschieden dicker, bei $293^{\circ} \mathrm{K}$ getemperter Ge-Filme bei $77^{\circ} \mathrm{K}$.

9 In die Abb. 7, 8 und 9 sind auch einige Versuche aufgenommen worden, bei denen die Filme vor der Sauerstoffeinwirkung Kohlenmonoxyd adsorbiert hatten. Wie in einer folgenden Arbeit (vergleiche Zitat ${ }^{2}$ ) über die getemperten Filmen (Abb. 5 und 6) - eine deutliche Widerstandsabnahme. Während die Widerstandszunahmen durchweg spontan verlaufen, sind die Widerstandsabnahmen mit einem Zeitbedarf verbunden. Das Austrittspotential ändert sich bei geringen Belegungen zunächst wenig und steigt bei höheren Belegungen um 0,1 bis 0,2 V. an.

Die Abhängigkeit der Effekte von der Belegung wird durch Abb. 7, 8 und 9 deutlich gemacht, in die auch Versuche mit weiteren Filmen anderer Schichtdicke aufgenommen sind. Man erkennt, daß Filme geringerer Schichtdicke eine stärkere Erhöhung ihres Widerstandes erleiden ${ }^{9}$.

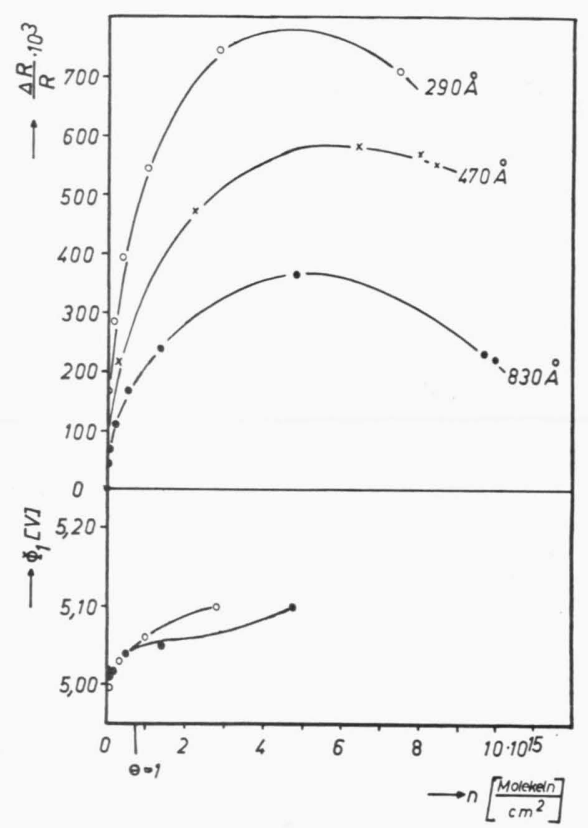

Abb. 9. Abhängigkeit der relativen Widerstandsänderung $\Delta R / R$ und des Austrittspotentials $\Phi_{1}$ verschieden dicker, bei $373^{\circ} \mathrm{K}$ getemperter Ge-Filme bei $273^{\circ} \mathrm{K}$.

\section{Deutung der Ergebnisse}

Da die an der reinen Oberfläche eines Ge-Kristalls befindlichen Ge-Atome eine oder zwei ihrer Tetraeder-Valenzbindungen nicht betätigen können, haben sie das Bestreben, zur Absättigung ihrer freien Bindungen Elektronen aus dem Inneren aufzunehmen (Abb. 10). Die hierdurch an der Oberfläche entstehenden Elektronenzustände bezeichnet man als

elektronische Wechselwirkung zwischen CO und Ge-Filmen gezeigt wird, ist die Vorbelegung mit CO ohne Einfluß auf die Sauerstoffeinwirkung. 
"TAмm-Zustände“. Durch den Elektronenentzug baut sich unterhalb der Oberfläche eine p-Randschicht auf (vgl. Abb. 1), deren Dicke mit etwa $1000 \AA$ angegeben wird. Die Dicke der verwendeten Filme liegt also völlig innerhalb der p-Randschicht.

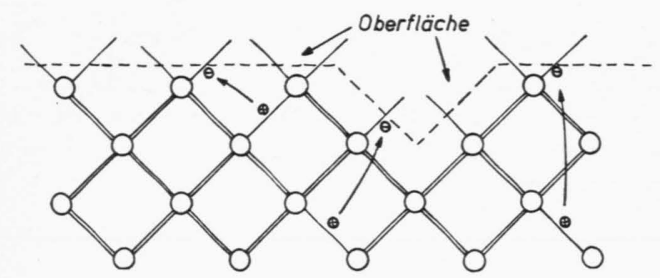

Abb. 10. Modell der freien Ge-Oberfläche nach HandLER ${ }^{10}$.

An mit Fremdmolekeln belegten Ge-Oberflächen findet man zwei Arten von Oberflächenzuständen, „fast states“ und „slow states“. Die fast states sind identisch mit den für die Photoelektronenemission reiner Ge-Oberflächen maßgebenden „Oberflächen( „ТАмм“-) zuständen“, die slow states hingegen werden z. B. bei der Chemisorption von Sauerstoff gebildet und sind in der Adsorptionsschicht („BSchicht") lokalisiert.

Die bei geringen Belegungen ohne Zeitbedarf erfolgenden Widerstandszunahmen (Abb. 4 bis 9) sind darauf zurückzuführen, daß der an der Oberfläche des Films in Atome dissoziierte Sauerstoff von den obersten Ge-Atomen gebunden wird. Hierdurch werden die bei dem unbelegten Film unter Aufbau der p-Randschicht an die TAMm-Zustände gelangten Elektronen wieder frei und können mit den Defektelektronen der p-Schicht rekombinieren. Dadurch geht die Aufwölbung des Valenzbandes zurück, das Diffusionspotential $V_{\text {D }}$ nimmt ab (Abb. 11 im Vergleich zu Abb.1). Durch den Aufbau der Adsorptionsschicht entsteht ein weiterer Potentialsprung $V_{\mathrm{B}}$ (vgl. Anm. ${ }^{11}$ ).

Bei ungeordneten Filmen überwiegt bei weitem die Widerstandszunahme infolge der Sauerstoffadsorption wegen der hohen Konzentration der unabgesättigten Bindungen. Bei stärker geordneten Filmen hingegen ist die Konzentration dieser Bindungen kleiner;

10 P. Handler, in R. H. Kingston, Semiconductor Surface Physics, University of Pennsylvania Press, Philadelphia 1957, S. 23.

11 W. H. Brattain u. J. Bardeen, Bell. Syst. Tech. J. 32, 1 [1953].

12 Das Durchlaufen eines Minimums der Oberflächenleitfähig. keit von Ge-Einkristallen bei der Adsorption von Sauerstoff wird auch von SPARnaAy und van RULER ${ }^{13}$ beschrieben. sie sind also bei geringerer Sauerstoffadsorption bereits gesättigt, so daß weitere hinzukommende $\mathrm{O}$ Atome als Elektronenakzeptoren wirken und erneut Defektelektronen unterhalb der Oberfläche erzeugen, so daß der Filmwiderstand wieder abnimmt ${ }^{12}$ (Abb. 8 und 9). Diese Abnahme ist besonders bei $77^{\circ} \mathrm{K}$ (Abb.5) sowie bei stärkerer Belegung mit einem größeren Zeitbedarf verbunden, da hier offensichtlich Diffusionsprozesse eine größere Rolle spielen.

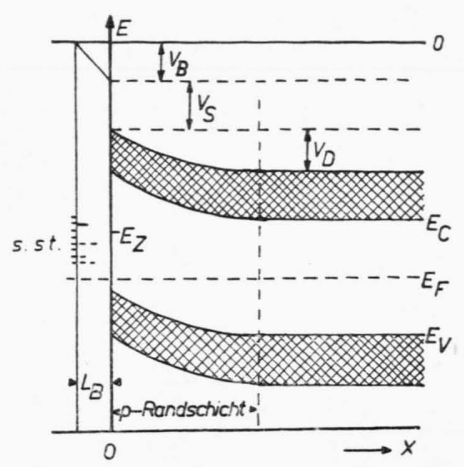

Abb. 11. Bändermodell der Ge-Oberfläche nach Chemisorption einer Oberflächenschicht der Dicke $L_{\mathrm{B}}$ (nach BARDEEN und Brattain ${ }^{11}$ ). Legende s. Abb. $1, V_{\mathrm{B}}$ Potentialsprung an der Adsorptionsschicht, s. st. = slow states.

Der Einfluß des Ordnungseffektes ist nicht nur auf die Abnahme der Zahl unabgesättigter Bindungen beim Ordnungsvorgang, sondern auch auf das gleichzeitige Abnehmen des Rauhigkeitsfaktors $f$ zurückzuführen, d. h. des Verhältnisses von wahrer zu geometrischer Filmoberfläche. Bei $293{ }^{\circ} \mathrm{K}$ geordnete Filme dürften einen $f$-Wert von etwa 2,5 , bei $373{ }^{\circ} \mathrm{K}$ geordnete von etwa 2 haben; ungeordnete Filme weisen eine bedeutend rauhere Oberfläche auf mit etwa ${ }^{14} f=6$.

In der freien Oberfläche sind die Dichten der Oberflächenatome pro $\mathrm{cm}^{2}$ und die Konzentrationen der ungepaarten Bindungen für die drei häufigsten Kristallflächen ${ }^{15}$

$\{100\} 0,62 \cdot 10^{15}$ Atome $/ \mathrm{cm}^{2}$ und $1,25 \cdot 10^{15}$ Bindungen $/ \mathrm{cm}^{2}$,

13 M. J. Sparnaay u. J. van Ruler, Physica 27, 153 [1961].

14 Diese Werte sind abgeschätzt aus Literaturwerten ${ }^{11}$ 15, 16 und früheren eigenen Messungen ${ }^{17}$ an Metallen.

15 M. Green, J. A. Kafalas u. P. H. Robinson, in R. H. Kingston, Semiconductor Surface Physics, University of Pennsylvania Press, Philadelphia 1957, S. 349.

16 Y.L. Sandler u. M. Gazith, J. Phys. Chem. 63, 1095 [1959].

17 R. Suhrmann, G. KRüGer u. G. Wedler, Z. phys. Chem., N. F. 30, 1 [1961]. 
$\{110\} \quad 0,88 \cdot 10^{15}$ Atome $/ \mathrm{cm}^{2}$ und $0,88 \cdot 10^{15}$ Bindungen $/ \mathrm{cm}^{2}$,

$\{111\} 0,72 \cdot 10^{15}$ Atome $/ \mathrm{cm}^{2}$ und $0,72 \cdot 10^{15}$ Bindungen $/ \mathrm{cm}^{2}$.

Man wird also mit etwa $0,74 \cdot 10^{15}$ Atomen und $1 \cdot 10^{15}$ Bindungen pro $\mathrm{cm}^{2}$ Mikrooberfläche rechnen können, von denen nach SHockLey ${ }^{18}$ etwa die Hälfte mit Elektronen aus dem Inneren besetzt sein sollten.

Bei ungeordneten Filmen (Abb. 7) ist die Widerstandszunahme bei etwa $n=2,5 \cdot 10^{15} \quad \mathrm{O}_{2}$-Molekeln pro $\mathrm{cm}^{2}$ Makro-Oberfläche abgeschlossen, entsprechend etwa $0,8 \cdot 10^{15}$ O-Atomen pro $\mathrm{cm}^{2}$ Mikrooberfläche. Die Adsorption ist bei diesem Belegungs$\operatorname{grad}(\Theta \approx 1 \quad 0$-Atome pro Ge-Oberflächenatom) noch völlig irreversibel (Abb.4). Das Austrittspotential ist dabei nur um $0,03 \mathrm{~V}$ angestiegen: Die Abnahme von $V_{\mathrm{D}}$ und das Auftreten von $V_{\mathrm{B}}$ lassen also das Austrittspotential nahezu unverändert. Erst bei höheren Belegungen (zwischen $\Theta=1$ und $\Theta=2$ ) führt die Zunahme von $V_{\mathrm{B}}$ zu einem etwas größeren Wert von $\Phi$. Die Zunahme von $\Phi$ beträgt jedoch nur etwa den zehnten Teil von der bei der Adsorption von $\mathrm{O}$-Atomen an Metallen im allgemeinen beobachteten. Eine reversible Adsorption (D in Abb. 4) tritt erst oberhalb $n=9,8 \cdot 10^{15} \mathrm{O}_{2}$ Molekeln pro $\mathrm{cm}^{2}$ Makrooberfläche auf, entsprechend $\Theta \approx 4,5$ O-Atomen pro Ge-Oberflächenatom.

Bei den bei $293{ }^{\circ} \mathrm{K}$ geordneten und bei $77^{\circ} \mathrm{K}$ untersuchten Filmen liegt das oben erwähnte Widerstandsmaximum (Abb. 8) im Mittel bei einer Belegung von $0,75 \cdot 10^{15} \mathrm{O}_{2}$-Molekeln pro $\mathrm{cm}^{2}$ MakroOberfläche, entsprechend einem Wert von $\Theta \approx 0,8$ O-Atomen pro Ge-Oberflächenatom. Im Gegensatz zu den ungeordneten Filmen hat bei diesem $\Theta$-Wert das Austrittspotential bereits die Hälfte seines Gesamtanstieges erreicht. In diesem Fall nimmt $V_{\mathrm{D}}$ nach dem Widerstandsmaximum wieder zu, weil die Randschicht jetzt Elektronen an die B-Schicht abgibt. Eine reversible Adsorption (D in Abb. 5 c) ist erst oberhalb einer Belegung von $n=5 \cdot 10^{15} \quad \mathrm{O}_{2}$-Molekeln pro $\mathrm{cm}^{2}$ Makrooberfläche $(\Theta \approx 5,3$ O-Atomen pro Ge-Oberflächenatom) zu beobachten.

Das Verhalten der bei $373{ }^{\circ} \mathrm{K}$ geordneten und bei $273{ }^{\circ} \mathrm{K}$ untersuchten Filme ähnelt weitgehend dem der bei $293{ }^{\circ} \mathrm{K}$ geordneten und bei $77^{\circ} \mathrm{K}$ untersuchten. Das Widerstandsmaximum (Abb.9) tritt jedoch erst bei $n \approx 5 \cdot 10^{15} \quad \mathrm{O}_{2}$-Molekeln pro $\mathrm{cm}^{2}$

18 W. Shockley, Phys. Rev. 56, 317 [1939].
Makro-Oberfläche $(\Theta=6,7$ O-Atome pro Ge-Oberflächenatom) auf. Das Austrittspotential, das sich zu Beginn der Belegung mit Sauerstoff nicht ändert, erreicht bei $\Theta \approx 1$ etwa wieder die Hälfte seines Gesamtanstieges. Eine reversible Belegung macht sich erst oberhalb von $n=9,7 \cdot 10^{15} \mathrm{O}_{2}$-Molekeln pro $\mathrm{cm}^{2}$ Makrooberfläche $(\Theta \approx 13)$ bemerkbar (Abb. 6, Punkt D). Die hohen $\Theta$-Werte deuten darauf hin, daß die Ge-Filme bei $273{ }^{\circ} \mathrm{K}$ bereits teilweise oxydiert werden. Diese Annahme wird dadurch gestützt, daß die kalorimetrisch bestimmten Adsorptionswärmen von $130 \mathrm{kcal} / \mathrm{Mol}^{19}$ in der Größenordnung der Bildungsenthalpie der Germaniumoxyde liegen.

Beim Vergleich der Abb. 7, 8, 9 fällt auf, daß der Widerstandsanstieg bei den ungeordneten (Abb. 7) und niedrig geordneten Filmen (Abb. 8) zu Anfang stärker ist als bei den höher geordneten (Abb. 9). Dies mag damit im Zusammenhang stehen, daß jedes Ge-Oberflächenatom in den Flächen $\{100\}$ zwei unabgesättigte Valenzen, in den Flächen $\{110\}$ und $\{111\}$ dagegen nur eine unabgesättigte Valenz hat. Bei der Bildung von $\mathrm{GeO}$ sollte also eine Reak-

\begin{tabular}{|c|c|c|c|c|}
\hline $\begin{array}{l}\text { Gas- } \\
\text { zugabe }\end{array}$ & $\begin{array}{c}n_{\text {irr. }} \\
\mathrm{Mol} / \mathrm{cm}^{2}\end{array}$ & $\begin{array}{c}\Phi_{1} \\
{[V]}\end{array}$ & $\begin{array}{c}\Phi_{2} \\
{[\mathrm{~V}]}\end{array}$ & $\begin{array}{l}\Delta \Phi \\
{[\mathrm{V}]}\end{array}$ \\
\hline \multicolumn{5}{|c|}{$\begin{array}{l}\text { an einem ungeordnetem Film von } 350 \AA \text { Schicht- } \\
\text { dicke, bei } 77^{\circ} \mathrm{K} \text { gemessen, }\end{array}$} \\
\hline 0 & 0 & 4,98 & 5,15 & 0,17 \\
\hline 1 & $3,23 \cdot 10^{13}$ & 4,97 & 5,16 & 0,19 \\
\hline 2 & $1,01 \cdot 10^{14}$ & 4,98 & 5,14 & 0,16 \\
\hline 3 & $2,06 \cdot 10^{14}$ & 4,97 & 5,18 & 0,21 \\
\hline 4 & $7,89 \cdot 10^{14}$ & 4,98 & 5,02 & $(0,04)$ \\
\hline 5 & $1,84 \cdot 10^{15}$ & 5,00 & 5,15 & 0,15 \\
\hline 6 & $5,72 \cdot 10^{15}$ & 5,09 & 5,25 & 0,16 \\
\hline \multicolumn{5}{|c|}{$\begin{array}{l}\text { an einem bei } 293^{\circ} \mathrm{K} \text { geordneten Film von } \\
880 \AA \text { Schichtdicke, bei } 77^{\circ} \mathrm{K} \text { gemessen. }\end{array}$} \\
\hline 0 & 0 & 4,98 & 5,11 & 0,13 \\
\hline 1 & $1,62 \cdot 10^{14}$ & 5,04 & 5,11 & 0,07 \\
\hline 2 & $8,08 \cdot 10^{14}$ & 5,09 & - & - \\
\hline \multicolumn{5}{|c|}{$\begin{array}{l}\text { an einem bei } 273^{\circ} \mathrm{K} \text { geordneten Film von } \\
830 \AA \text { Schichtdicke, bei } 273^{\circ} \mathrm{K} \text { gemessen. }\end{array}$} \\
\hline 0 & 0 & 5,02 & 5,05 & 0,03 \\
\hline 1 & $4,04 \cdot 10^{13}$ & 5,02 & 5,05 & 0,03 \\
\hline 2 & $9,29 \cdot 10^{13}$ & 5,01 & 5,20 & 0,19 \\
\hline 3 & $2,14 \cdot 10^{14}$ & 5,02 & 5,24 & 0,22 \\
\hline 4 & $5,13 \cdot 10^{14}$ & 5,04 & 5,06 & 0,02 \\
\hline 5 & $1,40 \cdot 10^{15}$ & 5,05 & 5,14 & 0,09 \\
\hline 6 & $4,80 \cdot 10^{15}$ & 5,10 & - & - \\
\hline
\end{tabular}

Tab. 1. Sauerstoffadsorption.

19 D. Brennan, D. O. Hayward u. B. M. W. Trapnell, J. Phys. Chem. Solids 14, 117 [1960]. 
tion an den $\{100\}$-Flächen einen doppelt so großen Widerstandseffekt haben wie eine Reaktion an den beiden anderen Flächen. Beim Ordnungsvorgang werden zuerst die instabileren Flächen $\{100\}$ abnehmen, an denen der größere Widerstandseffekt bei der Adsorption des Sauerstoffs zu erwarten ist. Auch die Adsorptionsgeschwindigkeit ${ }^{20}$ von Sauerstoff ist an den $\{100\}$-Flächen 10 - bis 20 mal größer als an den $\{111\}$-Flächen.

Nach Literaturangaben ${ }^{21}$ ist die Dichte der „slow states" bedeutend geringer $\left(10^{11}\right.$ bis $10^{12}$ pro $\left.\mathrm{cm}^{2}\right)$ als die der "Tамm-Zustände“ $\left(10^{15}\right.$ pro $\left.\mathrm{cm}^{2}\right)$. Die Emission der Photoelektronen sollte daher in über-

20 R. E. Schlier u. H. E. Farnsworth, J. Chem. Phys. 30, 917 [1959]. wiegendem Maße auch nach der Sauerstoffadsorption von den TAмm-Zuständen aus erfolgen. Diese Annahme wird dadurch gestützt, daß auch die mit Sauerstoff belegten Filme - wie die unbelegten zwei Austrittspotentiale $\Phi_{1}$ und $\Phi_{2}$ aufweisen und daß deren Differenz $\Delta \Phi$ (Tab. 1) im wesentlichen unabhängig von der Belegung ist. Die auftretenden Streuungen bei $\Phi_{2}$ und damit bei $\Delta \Phi$ sind auf die Schwierigkeiten der Bestimmung von $\Phi_{2}$ zurückzuführen ${ }^{3}$.

Die Untersuchungen wurden in dankenswerter Weise unterstützt von der Deutschen Forschungsgemeinschaft und dem Verband der Chemischen Industrie.
21 H. Statz, G. A. de Mars, L. Davis Jr. u. A. Adams JR., Phys. Rev. 101, 1272 [1956].

\title{
Der Einfluß von Druck auf die Supraleitung von Rhenium
}

\author{
Von J. L. Olsen, K. Andres, H. Meier und H. de Salaberry \\ Aus dem Institut für kalorische Apparate und Kältetechnik, \\ Eidgenössische Technische Hochschule, Zürich \\ (Z. Naturforschg. 18 a, 125-129 [1963] ; eingegangen am 13. Dezember 1962)
}

Herrn Professor Dr. Klaus Cuusius zum 60. Geburtstag gewidmet

\begin{abstract}
Der Einfluß von Druck $p$ auf die Sprungtemperatur $T_{\mathrm{c}}$ von Rhenium wurde gemessen. Man erhält $\partial T_{\mathrm{c}} / \partial p=-1,7 \pm 0,9 \cdot 10^{-12} \mathrm{Grad} \mathrm{dyn}^{-1} \mathrm{~cm}^{2}$. Dieses Resultat zusammen mit den Messungen von Andres über den Wärmeausdehnungskoeffizienten von Rhenium zwischen 0 und $10{ }^{\circ} \mathrm{K}$ wird benützt, um $\partial H_{\mathrm{c}} / \partial p$ als Funktion der Temperatur zu berechnen. Wir finden

$\left(\partial H_{\mathrm{c}} / \partial p\right)=-\left[2,7+1,3\left(T / T_{\mathrm{c}}\right)^{2}\right] \cdot 10^{-10}$ Gauss dyn ${ }^{-1} \mathrm{~cm}^{2}$.

Resultate über die Druckkoeffizienten der Sprungtemperatur in supraleitenden Elementen werden zusammengefaßt. Es wird gezeigt, daß sie eine Bestätigung des Unterschiedes zwischen den Mechanismen der Supraleitung in Übergangs- und Nicht-Übergangsmetallen geben.
\end{abstract}

Chusius hat vor mehr als 30 Jahren auf den $\mathrm{Zu}$ sammenhang zwischen Supraleitung und Atomvolumen hingewiesen. Das bekannte Diagramm, worin Cuusius ${ }^{1}$ die Lage der Supraleiter in der Atomvolumen-Atomgewichtsebene aufzeichnete und womit er zeigen konnte, daß nur die Elemente, deren atomare Volumina in gewissen Grenzen lagen, supraleitend sind, ist für mehr als eine Generation von Tieftemperaturphysikern ein Ansporn zur Untersuchung des Einflusses des Atomvolumens auf die Sprungtemperatur von Supraleitern geblieben.

Zur Zeit sind Messungen des Einflusses des Atomvolumens auf die supraleitenden Eigenschaften eines

1 K. Cuusius, Z. Elektrochem. 38, 312 [1932].

2 T. H. Geballe, B. T. Matthias, G.W. Hull u. E. Corenzwit, Phys. Rev. Letters 6, 275 [1961].
Metalles von besonderem Interesse, weil sie den kürzlich von Geballe, Matthias, Hull und CorenzwIT $^{2}$ entdeckten Unterschied zwischen Supraleitung in Übergangsmetallen und in Nicht-Übergangsmetallen bestätigten. Das von diesen Autoren beobachtete Fehlen eines Isotopeneffektes in der Supraleitung von Ruthenium ${ }^{2}$ und Osmium ${ }^{3}$, sowie der anomale Isotopeneffekt in Molybdän ${ }^{4}$ weisen darauf hin, daß die Elektron-Phonon-Wechselwirkung, die normalerweise als Ursache der Supraleitung in Nicht-Übergangsmetallen betrachtet wird, in diesen Metallen entweder fehlt oder stark verändert ist. Der Mechanismus der Supraleitung in Übergangsmetallen ist

3 T. H. Geballe u. B. T. Matthias, IBM J. Research Develop. 6, 256 [1962].

4 T. H. Geballe u. B. T. Matthias, Proc. 8th Int. Conf. Low Temp. Physics, 1962. 